\title{
Examining the efficacy of anti-predator training for increasing survival in conservation translocations: a systematic review protocol
}

\author{
Alison L. Greggor ${ }^{1 *}$, Catherine J. Price ${ }^{2}$ and Debra M. Shier ${ }^{1}$
}

\begin{abstract}
Background: How animals respond to predators can have consequences when they are reintroduced into the wild or translocated to new habitats. Animals raised in captivity often lack adequate experience with predators, and wild animals can be ill-equipped to respond to invasive predators. When these animals are released or translocated for conservation purposes, their naivety can jeopardize their survival and the outcome of the conservation intervention. Anti-predator training, i.e. the purposeful exposure of animals to predators or predatory-like cues for promoting predatory learning and awareness, is often suggested to be a useful tool in combating prey naivety. However, the prevalence of such training and the evidence for its effectiveness in conservation settings are currently unknown. We detail a set of protocols aimed at resolving both of these unknowns.

Methods: We will aim to gather studies from multiple databases and grey literature sources which document the occurrence of anti-predator training. We will search beyond the conservation management literature to also cover interventions aimed at promoting anti-predator behaviour in commercial contexts and other academic fields (e.g. animal cognition, behavioral ecology). Studies will be screened in two phases. The first stage of screening will collect studies that conduct anti-predator training. Metadata from this stage will help highlight biases in the use of antipredator training across geographic locations, funding contexts and taxonomic groups. We will then further screen for research that measures training efficacy either by using learning assessments, designating experimental groups, or by collecting post-release survival data. A narrative synthesis at this stage will describe the relative proportion of studies that measure the efficacy of their training. The smaller research pool will then be systematically reviewed to assess the efficacy of anti-predator training. We will attempt to extract data from all studies which assess efficacy, judging study validity and conducting a meta-analysis if sufficient evidence is found. By creating two stages to our screening and review of evidence, we will be able to better judge the biases and reliability of the efficacy evidence we find.
\end{abstract}

Keywords: Conservation behaviour, Pre-release training, Species reintroduction, Species translocation

\section{Background}

The need to mitigate prey naivety in species translocations Conservation translocations are last-resort tools used in species recovery plans and are often not enacted unless prior conservation efforts have failed [1,2]. Translocations of non-threatened animal species are also becoming increasingly common for habitat restoration purposes

\footnotetext{
*Correspondence: AGreggor@sandiegozoo.org

${ }^{1}$ Institute for Conservation Research, San Diego Zoo Global, Escondido,

CA, USA

Full list of author information is available at the end of the article
}

[3]. Overall, conservation translocations encompass dedicated transfers of animal species for purposes ranging from mitigating dwindling populations, to establishing a previously extirpated species, to expanding the range of an existing species $[4,5]$. Despite a growing number of successes, conservation translocations often fail [6]. A significant barrier to success is that animals bred or transferred from captivity often lack relevant survival skills for life in their new habitat [1, 7, 8]. Additionally, even wild-born animals can lack sufficient defences against introduced or invasive predators, post translocation [9], especially if founder groups are composed of

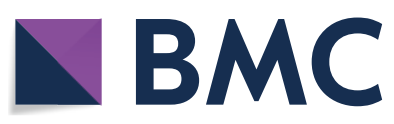

(c) The Author(s) 2019. This article is distributed under the terms of the Creative Commons Attribution 4.0 International License (http://creativecommons.org/licenses/by/4.0/), which permits unrestricted use, distribution, and reproduction in any medium, provided you give appropriate credit to the original author(s) and the source, provide a link to the Creative Commons license, and indicate if changes were made. The Creative Commons Public Domain Dedication waiver (http://creativecommons.org/ publicdomain/zero/1.0/) applies to the data made available in this article, unless otherwise stated. 
juveniles [10]. Therefore, training enacted prior to releasing animals has the potential to equip animals with skills they would otherwise lack.

One of the essential skills that is known to deteriorate in captivity is anti-predator behaviour [7, 11, 12]. Predation is considered to be one of the biggest risks to survival post-release, and can be major source of translocation failure [13]. Since optimal anti-predator behaviour is acquired through learning in many species [14-16], efforts to train animals to recognize and respond to native or invasive predators prior to release has much appeal. Despite there being examples where anti-predator training has been shown to improve conservation translocation outcomes (e.g. [17]), it is currently unclear whether the benefits of anti-predator training can be generalized across species and contexts. Additionally, best-practice standards for conducting such training do not exist, and resolving the details of training which contribute to its effectiveness has been highlighted as a high priority conservation behaviour issue [18].

Much of the theory involved in developing anti-predator training comes from laboratory-based psychological studies. From an academic standpoint, the study of anti-predator learning has been of interest for revealing insight into associative learning mechanisms [19], and predator-based cognitive biases [20]. However, there has been comparably less research into the application of these mechanisms in conservation settings. The lack of broad evidence in favour of conducting anti-predator training is symptomatic of the fact that studies in reintroduction biology rarely test a priori hypotheses in the process of interventions [21]. For species recovery teams that manage translocation protocols, measuring the survival benefits of the training can be difficult to justify because it can require creating a control group that is witheld from the training, or a group that receives an alternative training regime. For example, there are ethical considerations to be made when potentially depriving individuals of an endangered species of training that could increase their chances of survival. Additionally, species recovery teams often work with small numbers of individuals, and may be unable to divert time or resources towards research at the cost of pressing management actions. Thus, better evidence for the success of anti-predator training may need to come from parallel fields, semi-wild animals, or from surrogate species that are not at risk of extinction. For instance, refinement of anti-predator training is of great interest to the commercial fishing and hunting industries. The management of many commercial and recreational fish populations rely heavily on recruitment from stocks of hatchery-reared fish, which tend to suffer large losses soon after release [22], due to a lack of adequate foraging and anti-predator behaviour
[23]. Therefore, similar practices with non-threatened species and in non-conservation settings are likely to provide highly relevant evidence for conservation translocations, depending upon their training design.

There are several ways to design anti-predator training, but all rely on the premise that exposing animals to a real or simulated predator will invoke the species' evolved learning processes if the exposure is sufficiently aversive [14]. To help create aversion, often these exposures are paired with conspecific alarm cues, such as chemical alarm signals or auditory alarm calls, or paired with other aversive stimuli, such as electric stimulation, or startling flashes of light. Training can also present a predatory experience, such as a simulated predation event, which can differ in severity. For example, training regimes can involve the presentation of a dead conspecific alongside a predator (similar set up to [24]), or can present a predator actually attacking an animal [25]. Optimal training stimuli must be sufficiently aversive to elicit effective anti-predator behaviour, or risks a poor training outcome and compromised welfare following release [7]. Because there are numerous ways to simulate a predator or attack, it is currently unclear how close the simulation needs to be to an actual attack to be effective [18]. Additionally, there are a number of other details that could influence training effectiveness, which have not been explored systematically, such as the timing of training in a species development, the type of cue naturally produced for antipredator learning, or the evolutionary history of the prey species.

The effectiveness of training can theoretically be measured in several ways. A direct measure of effectiveness examines the ultimate survival value of the training as an intervention (e.g. [17]). In order to measure the survival benefits of training, animals must be split into experimental groups. Either a control group of animals must forego the training, or each group receives a different type of training. After release, the rate of survival can be compared between the experimental groups. In cases where practitioners are unable to measure the efficacy of training post-release, the documentation of predatory learning may serve as a potential proxy for the success of anti-predator training (e.g. [26]); although the link between the two has not been validated for many species. Training regimes that include a baseline assessment and a post-training assessment of anti-predator responses (or related behaviors, e.g. [27]) are necessary for providing evidence that the training increased anti-predator responses via learning. In order to demonstrate learning, the post-training assessment would need to occur after animals have calmed down from any heightened stress response caused by exposure to the training. Ideally this method would also consider a control group, thereby 
fulfilling a BACI (Before-After-Control-Intervention) design. Despite there being multiple ways to measure training efficacy, it is currently unclear how many training programs actually attempt to measure the success of their training.

\section{Topic identification and stakeholder engagement}

Greater investigation into the effectiveness of anti-predator training was identified as a priority research issue during a workshop with conservation managers and conservation behaviour researchers (see [18] for a description of the workshop, diversity of participants, and the resulting list of research priorities). A consultation process about anti-predator training then ensued between the authors and various stakeholders via email and inperson meetings. Stakeholders were identified as those who either engage in anti-predator training or advise on endangered species' management, such as permitting agencies, land-use partners, and species recovery teams. Consulted stakeholders included members of the U.S. Fish and Wildlife Service, Hawaii Department of Land and Natural Resources, Zoological Society of London, and the U.S. Geological Survey. After initial consultation it was decided that a traditional review would not be sufficient to adequately survey the diverse literature sources where anti-predator training is often documented. A formal evidence synthesis is necessary for determining the efficacy and best-practice for anti-predator training because the academic, peer-reviewed literature alone on this topic is likely to be highly biased towards positive results and successful translocations [28, 29]. Also, unless anti-predator training was a strong focus of release efforts, it may not be included in general publications about releases. Additionally, reviews that focus only on the conservation applications of anti-predator training are likely to miss the laboratory-based literature and commercial applications which can equally assesses predatory learning and survival.

Once the need for a systematic evidence synthesis was realized, specific review objectives were developed by the authors, with guidance from stakeholders about the details of training most pertinent to their operations.

\section{Objective of the review}

Our efforts will first focus on gathering evidence about the context in which anti-predator training has been attempted, and where that training has involved an assessment of efficacy. This effort will allow for an informed discussion about what proportion and types of studies measure the efficacy of their training. The studies that do assess efficacy will then be pooled for a systematic review and meta-analysis to determine the factors that contribute to training success. Together, the two screening stages and subsequent review will allow for a thorough evaluation of the biases and reliability of the efficacy evidence we find. Our review objectives are laid out through primary and secondary questions that are formulated using PICO elements (Population-Intervention-Comparator-Outcome); a common approach in systematic reviews and maps, including those which evaluate animal behavior [30, 31].

Primary question: In which contexts, locations and species has anti-predator training been implemented?

The primary question will be used for the first stage of evidence screening. Elements of the primary question are:

Population Wild, wild-caught or captive-born vertebrate animals subject to anti-predator training, regardless of the animals' ultimate purpose (conservation, academic, recreational, or commercial).

Intervention Exposure to predators and/or predatorylike cues designed to increase predator recognition, detection and anti-predatory responses, or survival following release into the wild.

Secondary question: Under what conditions is prerelease anti-predator training effective in increasing predatory learning or survival in translocated animals?

The secondary question contains the same Population and Intervention elements as the primary question, but it additionally assesses relevance based on described Comparators and Outcomes. This secondary question will be used to select studies for the systematic review. The additional elements of the secondary question include:

Comparator (i) A comparison of anti-predator awareness or behaviour before and after training; and/or

(ii) A control or a comparison group of individuals which do not receive training, or which receive a different type of training.

Outcome (i) Behavioural measures demonstrating anti-predator learning during the training; and/or

(ii) Metrics of survival for individuals after release into the wild.

In order to be considered for the systematic review, studies must contain at least one of the comparators and one of the outcomes. 


\section{Methods}

\section{Searching for articles}

A scoping exercise was conducted using 15 known studies containing anti-predator training, five from each field where training is used (conservation management, industry, non-conservation academic fields) (see Additional file 1 for benchmark list). The exercise was used to determine whether the proposed search strategy adequately retrieved relevant literature, which it did for all 15 studies listed. Literature databases will be searched using Boolean operators (modified to the specifics of the database language) with institutional access from the University of Cambridge (alumni access) and from the University of California, Los Angeles. Only Population and Intervention terms will be searched to form the pool of studies from which Comparator and Outcome categories will later be assessed. The following search string is formatted for the ISI Web of Science database, and will be reformatted depending upon the preferred search language of each database.

Population (transloc* OR reintrodu* OR re-introduc* OR rewild* OR *release* OR conservation OR captiv* OR hatchery* OR relocat $\left.{ }^{*}\right)$

\section{AND}

Intervention (("predat* OR fear OR enemy) AND (condition* OR naïv* OR train* OR treatment OR exposure OR experience OR learn* OR recogn* OR avoid* OR cue OR stimul* OR defen $\$$ e))

\section{Bibliographic searches}

We will aim to collect studies from the following publication databases in both English and Spanish languages:

- ISI Web of Science: Core Collection, BIOSIS Citation Index and Zoological Record

- Scopus

- JSTOR

- Wiley Online Library

- APA PsychNet

- Academic Search Complete

- BioOne

- WildPro Electronic Library

- ProQuest

- ENDANGER, Deakin University, Australia

- Trove (https://trove.nla.gov.au)

- National ETD Portal: South African Theses and Dissertations
- EThOS: UK Theses and Dissertations.

\section{Search engines}

The following search engines and web sites will also be used for finding studies:

- Google Scholar (http://www.GoogleScholar.com).

- ResearchGate (http://www.researchgate.net).

Google Scholar will be searched through Google Chrome's "incognito" mode, and cookies and browser history will be cleared between searches. Search results will be sorted by relevance and only the first 50 results per search will be examined. ResearchGate will be used for finding articles already uploaded by authors and for making an open call for evidence (see below).

\section{Specialist websites}

The following specialist websites will be queried for species reports which contain anti-predator training

- IUCN general publications (https://portals.iucn.org/ library/dir/publications-list).

- IUCN Conservation Planning Specialist Group (http://www.cpsg.org/document-repository).

- Conservation Evidence (http://www.ConservationEvi dence.com).

- US Federal Science database (https://www.scien ce.gov/).

- Association of Zoos and Acquarium's (AZA) Animal Programs Database (https://www.aza.org/speciessurvival-plan-programs); Access through San Diego Zoo Global.

\section{Additional searches}

Other sources of species recovery plans will be sought via emails to US state and federal agencies (e.g. NOAA and USFWS), to ecological consultancy organizations (such as Island Conservation and Wildlife Management International) and to conservation breeding facilities worldwide which are known to engage in species translocations. The list of re-introduction practitioners compiled by the IUCN's Reintroduction Specialist Group (see [32]) will be consulted to make these contacts. Finally, open calls for research and reports of anti-predator training will be sought on online platforms such as Twitter, ResearchGate and through relevant email list servers such as ECOLOG-L.

All database searches conducted and methods of retrieval for grey literature will be documented and 
presented in a additional file for the final publication of the review (an example datasheet can be found as Additional file 2). Should the process of gathering and examining studies for the review take longer than 18 months, the searches will be re-run before the final analysis to collect recently published studies.

\section{Screening process}

The study screening process and measures of interscreener reliability will be conducted through the online, open source platform of CADIMA (https://www.cadim a.info/). CADIMA streamlines the creation of systematic evidence syntheses and allows for the easy removal of duplicate studies [33]. In the case where the same intervention is documented in a published study and a grey literature source, both will be consulted, but only the one with the best description of methods and results will be included. In this case, it will be noted that a duplicate existed from another source. If one source contains more information about one method and the other source contains more information about a second method used, then the two sources will be combined for assessing the metadata metrics. Review articles will not be included in the map or review, but their reference lists will be checked to ensure that all relevant literature they cover is included in our searches.

Studies will be screened at the title and abstract stage simultaneously because our scoping search of published articles showed that the article relevance could not be consistently assessed via the title alone. Prior to screening, reviewer consistency will be checked through the CADIMA software, and inclusion criteria clarified if needed. Subsequently, studies will be screened primarily by two reviewers at the abstract stage, who will overlap in their assessment of a randomly selected $10 \%$ of studies (up to a maximum of 100 studies). A third reviewer will then verify the reliability of the two reviewers by looking at an additional 100 studies of each reviewer. Interscreener reliability will be judged via Kappa calculations, with a value greater than 0.6 deemed to be acceptable [34, $35]$. In cases where reviewers come to conflicting conclusions about a given study, the study will be discussed and inclusion criterial will be modified if needed. In cases where there is uncertainty about the relevance of a study, the reviewers will lean towards inclusion. In cases where the authors' own studies are under consideration at any stage of the screening process, a third-party reviewer will be consulted, blind to the author list of the said study.

All studies identified as relevant at the abstract and title stage will proceed to full text assessments. Full texts will be retrieved via open source platforms and institutional access where possible. If the full text of a given study cannot be found through those means, authors will be contacted to request a copy. Reviewers will overlap in $10 \%$ of full text assessments, up to a maximum of 50 studies. A list of all studies excluded at the full-text stage will be compiled, along with the reason for their exclusion.

\section{Eligibility criteria}

Initially all studies will be included which contain the relevant Population and Intervention criteria.

- Eligible subjects: Non-human vertebrates that are either found in the wild, brought into captivity from the wild for training or head-starting, or that are bred in captivity. Captivity is defined as being housed in a man-made enclosure or structure (regardless of duration or size). While the training may take place in captivity, a later assessment of the training efficacy (if applicable) may take place outside of captivity.

- Intervention: Training which involves exposure to live predators, predatory cues or stimuli intended to mimic a predator. The intention of the exposure must be either to: (A) increase levels of predatory wariness, anti-predator responses, or behavioral indicators of wariness (e.g. increased burrowing or sheltering), or; (B) to investigate heterogeneity in the effect of the exposure on predatory wariness or antipredator responses.

Where studies are deemed to be eligible at the full text stage based on Population and Intervention criteria, they will also be assessed based on the presence of Comparator and Outcome criteria to determine the study's suitability to be included in the review.

- Comparator: The study must contain one of the following types of comparison.

- A before/after (BA) comparison that investigates how behaviour changes before and after the training.

- A control/intervention (CI) design which designates a control group of individuals which do not receive training, while an experimental group does, or two or more experimental groups which receive different types of predatory or non-predatory stimuli.

- A before/after, control/intervention (BACI), which combines the two prior study designs, potentially with a combination of the different outcomes listed below. 
- Outcomes: Studies which aim to determine the learning effects of training need to measure changes to anti-predator behaviour. Eligible behaviours include measures of predatory recognition or detection via species-specific fear responses or by proxy responses such as changes to general wariness in the presence of the predatory stimuli, or other behavioral indicators of wariness (e.g. burrowing or shelter seeking behaviour). Studies which investigate learning outcomes can take the form of BA, CI or BACI studies. In contrast, those which aim to measure the survival benefit of the training need to report metrics of survival for individuals after release into the wild. The time frame of this assessment is not a criterion for inclusion. For studies that measure survival, only CI comparisons are logical, given the irreversible nature of survival.

\section{Study validity assessment}

Studies that pass the Population and Intervention screening stage but do not pass the Comparator and Outcome stage will not proceed to further critical appraisal. Instead, their meta-data will be used to help create a narrative synthesis that compares the relative number of studies which come from conservation, industry, and other academic fields, as well as the taxonomic and geographic distribution of the evidence, and whether the effectiveness of the training was measured. This synthesis ahead of the review will provide the context about where evidence exists for anti-predator training interventions and what proportion of studies measure the efficacy of their training.

Those studies which measured efficacy via learning assessments and/or survival metrics will be subject to deeper critical appraisal and a full systematic review. The review will aim to determine whether anti-predator training is effective, and will explore the potential sources of heterogeneity, depending on how many studies are available for comparisons. The studies subject to review will be critically appraised based on the following: study design, number of animals per treatment group, independence of the data points, the measure of efficacy used, the time between the intervention and efficacy measurement, and whether hypothesis-testing statistics were used to compare treatment groups. Studies with a BACI design will be considered the most robust, with $\mathrm{CI}$ and BA following subsequently. The data points will be considered independent if the individual animals are only involved in one type of predator-related intervention during the study. Measures of efficacy include assessments of learning via behaviour, or survival rates, both of which may vary in the time between measurement and the intervention. Studies that demonstrate effectiveness of anti-predator training will be weighted more strongly the longer the time between measurement and intervention. Conversely, studies which fail to demonstrate effectiveness will be weighted more strongly the shorter the time between measurement and assessment. At least $10 \%$ of studies (up to a maximum of 50 ) will be critically appraised by more than one person to verify that the process is repeatable and reliable. If appraisers come to different conclusions on any given study, their discrepancies will be discussed and the metrics of validity will be clarified if needed.

\section{Data coding and extraction strategy}

Metadata will be collected on the following variables from all studies which fulfil the Population and Intervention criteria

- Bibliographic information

- Full citation

- Publication type

- Duration of study (years)

- Study context

- Geographic location (country, latitude, longitude, biome)

- Research area (conservation, industry, other academic field)

- Population characteristics

- Trained species' common and Latin name(s)

- Trained species' IUCN status

- Trained species' duration of time in captivity

- If captive bred, number of generations in captivity

- Conservation translocation type (if applicable: reintroduction, translocation to new site, translocation to site with existing population; categories based on [2])

- Predator and intervention characteristics

- Predator type used for training (native versus invasive, aerial versus terrestrial/aquatic, and ambush versus sit-and-wait)

- Predatory stimulus category (e.g. auditory, visual, live, etc.)

- Direct or indirect cue (i.e. direct experience, or social exposure available only) 
- Predatory stimulus description

- No. exposures to predator or predator-like stimuli

The following additional meta-data variables will be collected on studies which also fulfil the Comparator and Outcome criteria, leading to critical appraisal:

- Study design and comparator information

- Metric of efficacy (either none, learning-based or survival-based)

- If survival-based, are there additional predator species in the habitat?

- Study design (e.g. BA, CI, BACI)

- Type of control

- No. animals (populations) per experimental group

- Randomized assignment of experimental groups

- Effect modifiers analysed

- Independence of data

- Hypothesis testing statistics used?

- Outcome characteristics

- Deemed success by authors

- Interval between intervention and outcome.

A number of these metadata variables are being simultaneously collected by review teams working on systematic evidence syntheses of other conservation behaviour interventions. The meta-data for all studies assessed will be included as additional file in the final publication. A sample datasheet for the metadata can be found as Additional file 3.

We will attempt to extract data from all studies which assess efficacy. Should sufficient detail not be included in the study itself to calculate effect sizes, data will be sought by online data repositories and contact with lead and corresponding authors. All studies for which the data cannot be retrieved will be excluded from the final metaanalysis. All extracted data will be incorporated into a standardized spreadsheet which will record each study's design, statistics used, sample size, number of training sessions conducted, outcome means, and a measure of variation (i.e. standard error or deviation). This extracted data will be used alongside the information collected from the meta-date file to conduct analyses.

\section{Potential effect modifiers/reasons for heterogeneity}

Although anti-predator learning is taxonomically widespread [36], the efficacy of training may differ depending on the species involved. Additionally, since anti-predator behaviour can erode over generations in captivity $[7,11,12]$, the duration of time spent in captivity could influence the effectiveness of the training. There can also be contextual differences in how close a predator simulation needs to mirror an actual predator, a source of heterogeneity that is not well understood [18]. Finally, theory and evidence suggest that animals differ in their ability to recognize and respond to invasive versus native predators (see [37] for a review). Therefore, the efficacy of training may be influenced by the evolutionary relationship the trained species has with the target predator. The stakeholders that have been involved thus far have expressed additional interest in understanding how often training needs to be conducted in order to be effective, depending on the type of training deployed. One additional source of heterogeneity that should be acknowledged, but may not have sufficient sample sizes to be investigated fully, is the effect of individual personality variables on the efficacy of learning in training scenarios. Variation in personality is known to influence anti-predator responses [38], but the interaction between personality and learning about new predators is still an emerging discipline.

\section{Data synthesis and presentation}

Two narrative syntheses will be created. The first will cover all studies that fulfil the Population and Intervention criteria and the second will be generated from the studies included in the review. The initial narrative synthesis will contain descriptive statistics on general metadata areas, such as the training context (conservation management, other academic fields, industry), species, location, and whether efficacy was assessed. This first synthesis will provide the context for the evidence of efficacy that we gather, by identifying knowledge gaps and under-researched areas of the literature. The second, more detailed synthesis that covers studies considered for full review will examine a larger number of metadata variables. The number of studies which adhere to each of the three experimental designs will be reported (e.g. BA, $\mathrm{CI}, \mathrm{BACI})$. The mean and standard deviation in the time between intervention and testing will be reported, split into the different measures of efficacy, if possible.

Should a meta-analysis be possible with the studies that assess efficacy, then we will quantitatively examine and summarize the efficacy and benefits of anti-predator training, which may vary depending on the species and the method of implementation. We will run our metanalysis in R [39], using the metafor package [40] to interrogate the sources of heterogeneity as fixed effects (as explained in [41]). Only the sources of heterogeneity that have ample studies for testing hypotheses will be included. During the meta-analysis we will account for the quality of studies in weighting the evidence provided. 
Publication bias will be assessed based on the "trim and fill" method which uses funnel plots to detect bias (see [42]). If necessary, our conclusions will be adjusted based on the estimated presence and direction of bias.

The entirety of our methods adheres to the ROSES guidelines and follows CEE standards of best practice for systematic reviews (see Additional file 4 for ROSES checklist).

\section{Additional files}

Additional file 1. A list of 15 studies used to verify that the search strategy retrieved studies from academia, industry and conservation.

Additional file 2. The datasheet that will be used to log all searches made across databases.

Additional file 3. The datasheet that will be used for collecting meta-data on eligible studies. It has been reformatted, based on the template from [43].

Additional file 4. Our declaration and checklist of adherence to the ROSES guidelines.

\section{Authors' contributions}

ALG wrote the first draft of the paper. All authors contributed to the writing of the final draft and approved of its content. All authors read and approved the final manuscript.

\section{Author details}

1 Institute for Conservation Research, San Diego Zoo Global, Escondido, CA, USA. ${ }^{2}$ School of Life and Environmental Sciences, University of Sydney, Sydney, NSW, Australia.

\section{Acknowledgements}

We sincerely thank Biljana Macura for training A.L.G. and C.J.P. in the methods of systematic evidence synthesis, and for subsequent support in answering review questions. This training was made possible with support from the Jacob Blaustein Center for Scientific Cooperation, The Swiss Institute for Dryland Environmental \& Energy Research, the Mitrani Department of Desert Ecology, and Ben-Gurion University of the Negev. We also thank Christian Köhl for advice on using CADIMA, John Ewen and other stakeholders for feedback on ideas or the manuscript.

\section{Competing interests}

The authors declare no competing financial interests, but two of the authors ALG and DS engage in species reintroduction programs that use anti-predator training. In cases where the authors' own studies are under consideration at any stage of the screening process, a third-party reviewer will be consulted, blind to the author list of the said study.

\section{Availability of data and materials}

The only data used in the preparation of this manuscript is the scoping exercise of benchmark studies, which is included as an additional file to this article. The search history and metadata from the final review will be made publically available upon publication.

\section{Consent for publication}

Not applicable.

\section{Ethics approval and consent to participate}

This study does not report on or include research on human subjects, but does report on research conducted on animals by other entities. The authors are relying on the ethics approval process for existing published studies and grey literature. Studies which fail to report their permitting or ethics review process both in the paper and after author contact (if necessary) will be dropped from the review.

\section{Funding}

A.L.G is funded by a Clark endowed postdoctoral fellowship, gifted to San Diego Zoo Global. The donors played no role in the design of this study. Publication costs are funded by the Clark endowed postdoctoral fellowship and a collaboration between Ben Gurion University and Monash University.

\section{About this supplement}

This article has been published as part of Environmental Evidence Volume 8 Supplement 1, 2019: Using animal behavior in conservation management. The full contents of the supplement are available online at https://environmen talevidencejournal.biomedcentral.com/articles/supplements/volume-8-suppl ement-1

\section{Publisher's Note}

Springer Nature remains neutral with regard to jurisdictional claims in published maps and institutional affiliations.

Published: 13 June 2019

\section{References}

1. Seddon PJ, Armstrong DP, Maloney RF. Developing the science of reintroduction biology. Conserv Biol. 2007;21:303-12.

2. Ewen JG, Armstrong DP, Parker KA, Seddon PJ, editors. Reintroduction biology: integrating science and management (No. 9). Hoboken: Wiley; 2012.

3. Watson DM, Watson MJ. Wildlife restoration: mainstreaming translocations to keep common species common. Biol Conserv. 2015;191:830-8.

4. IUCN. Guidelines for reintroductions and other conservation translocations. Gland: IUCN/Species Survival Commission; 2012

5. Swaisgood RR, Ruiz-Miranda C. Moving animals in the right direction: making conservation translocation an effective tool. In: Koprowski J, Krausman P, editors. International wild life: contemporary challenges in a changing world. TheWildlife Society and Johns Hopkins University Press

6. Fischer J, Lindenmayer DB. An assessment of the published results of animal relocations. Biol Conserv. 2000;96:1-11.

7. Shier DM. Manipulating animal behavior to ensure reintroduction success. In: Berger-Tal O, Saltz D, editors. Conservation behavior: applying behavioral ecology to wild life conservation and management. Cambridge: Cambridge University Press; 2016. p. 275-304.

8. Tavecchia G, Viedma C, Martinez-Abrian A, Miguel-Angel B, Gomez JA Oro D. Maximizing re-introduction success: assessing the immediate cost of release in a threatened waterfowl. Biol Conserv. 2009;142:3005-12.

9. West R, Letnic M, Blumstein DT, Moseby KE. Predator exposure improves anti-predator responses in a threatened mammal. J Appl Ecol. 2017:55:147-56. https://doi.org/10.1111/1365-2664.12947

10. Shier DM. Effect of family support on the success of translocated blacktailed prairie dogs. Conserv Biol. 2006;20:1780-90.

11. Yoerg SI, Shier DM. Captive breeding and anti-predator behavior of the (Dipodomys heermanni) Heermann's kangaroo rat. Final Report. Sacramento; 2000.

12. Kraaijeveld-Smit FJL, Griffiths RA, Moore RD, Beebee TJC. Captive breeding and the fitness of reintroduced species: a test of the responses to predators in a threatened amphibian. J Appl Ecol. 2006;43:360-5.

13. Moseby KE, Read J, Paton D, Copely P, Hill B, Crisp H. Predation determines the outcome of 10 reintroduction attempts in arid South Australia. Biol Conserv. 2011;144:2863-72.

14. Griffin AS, Blumstein DT, Evans CS. Training captive-bred or translocated animals to avoid predators. Conserv Biol. 2000;14:1317-26.

15. Griffin AS. Social learning about predators: a review and prospectus. Learn Behav. 2004;32:131-40.

16. Brown C, Laland KN. Social learning and life skills training for hatchery reared fish.J Fish Biol. 2001:59:471-93.

17. van Heezik Y, Seddon PJ, Maloney RF. Helping reintroduced houbara bustards avoid predation: effective anti-predator training and the predictive 
value of pre-release behaviour. Anim Conserv. 1999;2:155-63. https://doi. org/10.1111/j.1469-1795.1999.tb00061.x.

18. Greggor AL, Berger-Tal O, Blumstein DT, Angeloni L, Bessa-Gomes C, Blackwell BF, et al. Research priorities from animal behaviour for maximising conservation progress. Trends Ecol Evol. 2016;31:953-64.

19. Griffin AS. Socially acquired predator avoidance: is it just classical conditioning? Brain Res Bull. 2008;76:264-71.

20. Cook M, Mineka S. Observational conditioning of fear to fear-relevant versus fear-irrelevant stimuli in rhesus monkeys. J Abnorm Psychol. 1989;98:448-59.

21. Taylor G, Canessa S, Clarke RH, Ingwersen D, Armstrong DP, Seddon PJ, et al. Is reintroduction biology an effective applied science? Trends Ecol Evol. 2017;32:873-80.

22. Wiley RW, Whaley RA, Satake JB, Fowden M. An evaluation of the potential for training trout in hatcheries to increase post-stocking survival in streams. North Am J Fish Manag. 1993;13:171-7.

23. Brown C, Day RL. The future of stock enhancements: lessons for hatchery practice from conservation biology. Fish Fish. 2002;3:79-94.

24. Kruuk $\mathrm{H}$. The biological function of gulls' attraction towards predators. Anim Behav. 1976;24:146-53.

25. White TH, Collazo JA, Vilella FJ. Survival of captive-reared Puerto Rican parrots released in the Caribbean National Forest. Condor. 2005;107:424-32.

26. Gonzalo A, López P, Martín J. Iberian green frog tadpoles may learn to recognize novel predators from chemical alarm cues of conspecifics. Anim Behav. 2007;74:447-53.

27. Moseby KE, Cameron A, Crisp HA. Can predator avoidance training improve reintroduction outcomes for the greater bilby in arid Australia? Anim Behav. 2012;83:1011-21. https://doi.org/10.1016/j.anbeh av.2012.01.023.

28. Jennions MD, Møller AP. Publication bias in ecology and evolution: an empirical assessment using the 'trim and fill' method. Biol Rev. 2002;1:211-22.

29. Miller KA, Bell TP, Germano JM. Understanding publication bias in reintroduction biology by assessing translocations of New Zealand's Herpetofauna. Conserv Biol. 2014;28:1045-56.

30. Berger-Tal O, Greggor AL, Macura B, Adams CA, Blumenthal A, Bouskila $A$, et al. Systematic reviews and maps as tools for applying behavioral ecology to management and policy. Behav Ecol. 2018. https://doi. org/10.1093/beheco/ary130/5123582.

31. James KL, Randall NP, Haddaway NR. A methodology for systematic mapping in environmental sciences. Environ Evid. 2016;5:7. https://doi. org/10.1186/s13750-016-0059-6

32. Soorae PS, Seddon PJ. Re-introduction practitioners directory. Gland: IUCN/SSC Re-introduction Specialist Group and the National Commission for Wildlife Conservation and Development (NCWCD); 1998.

33. Kohl C, McIntosh EJ, Unger S, Haddaway NR, Kecke S, Schiemann J, et al. Online tools supporting the conduct and reporting of systematic reviews and systematic maps: a case study on CADIMA and review of existing tools. Environ Evid. 2018;7:1-17. https://doi.org/10.1186/s1375 0-018-0115-5.

34. Cohen J. A coefficient of agreement for nomial scales. Educ Psychol Meas. 1960;20:37-46.

35. Edwards P, Clarke M, DiGuiseppi C, Pratap S, Roberts I, Wentz R. Identification of randomized controlled trials in systematic reviews: accuracy and reliability of screening records. Stat Med. 2002;21:1635-40.

36. Griffin AS. Social learning about predators: a review and prospectus. Anim Learn Behav. 2004;32:131-40. https://doi.org/10.3758/BF03196014.

37. Carthey AJR, Blumstein DT. Predicting predator recognition in a changing world. Trends Ecol Evol. 2017;33:106-15. https://doi.org/10.1016/j. tree.2017.10.009

38. Jones KA, Godin JGJ. Are fast explorers slow reactors? Linking personality type and anti-predator behavior. Proc R Soc B Biol Sci. 2010;277:625-32.

39. Team RDC. R: a language and environment for statistical computing. Vienna: R Foundation for Statistical Computing; 2015.

40. Viechtbauer W. Metafor: meta-analysis package for R (Version 1.9-5). 2015

41. Polanin JR, Hennessy EA, Tanner-Smith EE. A review of metaanalysis packages in R. J Educ Behav Stat. 2016;20:1-37. https://doi. org/10.3102/1076998616674315.

42. Duval S, Tweedie R. Trim and Fill: a simple funnel-plot-based method. Biometrics. 2000;56:455-63.

43. Snijders L, Greggor AL, Hilderink F, Doran C. Effectiveness of animal conditioning interventions in reducing human-wildlife conflict. A systematic map protocol. Environ Evid. (in press)
Ready to submit your research? Choose BMC and benefit from:

- fast, convenient online submission

- thorough peer review by experienced researchers in your field

- rapid publication on acceptance

- support for research data, including large and complex data types

- gold Open Access which fosters wider collaboration and increased citations

- maximum visibility for your research: over 100M website views per year

At BMC, research is always in progress.

Learn more biomedcentral.com/submissions 